Meta

Journal des tradlucteurs

Translators' Journal

\title{
La théorie de la traduction : à quoi ça sert ?
}

\section{Robert Larose et Denys Lessard}

Volume 30, numéro 4, décembre 1985

URI : https://id.erudit.org/iderudit/003406ar

DOI : https://doi.org/10.7202/003406ar

Aller au sommaire du numéro

Éditeur(s)

Les Presses de l'Université de Montréal

ISSN

0026-0452 (imprimé)

1492-1421 (numérique)

Découvrir la revue

Citer cette note

Larose, R. \& Lessard, D. (1985). La théorie de la traduction : à quoi ça sert? Meta, 30(4), 405-407. https://doi.org/10.7202/003406ar d'utilisation que vous pouvez consulter en ligne.

https://apropos.erudit.org/fr/usagers/politique-dutilisation/ 


\section{LA THÉORIE DE LA TRADUCTION : À QUOI CA SERT ?*}

Lors du VIIIe Congrès mondial de la Fédération internationale des traducteurs, $M$. Jean Darbelnet $(1977: 193)$ a émis l'opinion qu'" au niveau du premier cycle (de la formation), le cours sur la théorie de la traduction était d'une utilité contestatble, eu égard au temps et à l'énergie qu'il absorbait ". Cet éminent linguiste n'a cependant pas indiqué pourquoi l'enseignement de la théorie devait nécessairement être utile au sens pragmatique qu'il confêre probablement à ce mot. Et il n'a pas non plus expliqué pourquoi la mise à l'index d'un cours de réflexion sur la traduction devait procéder du temps et de l'énergie exigés par celui-ci, un peu comme si l'utilité d'un cours était en fonction inverse du volume d'efforts qu'on lui consacrait ! Enfin, un tel commentaire nous semble revenir à appliquer un critère d'efficience professionnelle à une activité qui se veut, justement, affranchie de contraintes de cet ordre, et il y aurait eu lieu de rendre compte d'un tel paradoxe.

L'examen des programmes de baccalauréat en traduction de cinq universités (Concordia, Laval, Montréal, Ottawa et Trois-Rivières) permet de constater que la théorie de la traduction est enseignée dans chacun d'entre eux, sauf sans doute à l'Université Laval où le cours « Pratique et théorie de la version ", qu'on nous a présenté comme un cours de théorie, n'en a pas moins pour objectif une " application des principes de la stylistique comparée à la traduction de l'anglais vers le français" ".

En ce qui concerne les quatre autres programmes, le seul aspect commun aux cours de théorie qu'ils contiennent est qu'ils sont donnés lors de la dernière année du baccalauréat1. Pour le reste, il existe une diversité de conception accusée. À l'Université de Montréal , les théories et l'histoire de la traduction sont réparties sur deux cours. À l'Université d'Ottawa, elles sont enseignées dans un cours unique. Dans d'autres cas, c'est une perspective syn- 
chronique qui a été adoptée, et un seul cours est offert (Université Concordia, UQTR).

Quoi qu'il en soit, l'essentiel est que l'on a reconnu, dans chaque institution, le besoin de justifier la réflexion sur la théorie, de faire connaître ses rapports avec la pratique, d'examiner les obstacles fondamentaux à la traduction (p.ex. la question de savoir si la traduction peut être totale) et de préciser la nature de certains concepts clés, comme celui de l'équivalence.

Les professeurs chargés d'enseigner de tels cours ne sont pas les seuls à trouver difficile de circonscrire et définir le concept de " théorie de la traduction ", et l'on ne sera pas surpris qu'il en aille de même pour les étudiants. Au début du cours de théorie offert à l'UQTR à la session d'automne 1984, il a été demandé à ces derniers $a$ ) pourquoi ils suivaient ce cours, et $b$ ) ce que signifiait pour eux le terme "théorie ". Les réactions et opinions exprimées à ce sujet n'ont pas manqué d'être variées.

En réponse à la première question, les étudiants ont indiqué qu'ils espéraient " accroître leurs connaissances générales ", "découvrir les différents auteurs et les différentes théories existantes au sujet de la traduction", "connaître les divers points de vue sur la manière de traduire ", "arriver à se faire une opinion sur ce qui constitue la meilleure façon de traduire ou de juger une traduction ", " faire un survol des grands traducteurs", et "perfectionner des connaissances sur la traduction, le métier et les méthodes ". Quant au mot " théorie ", il s'est trouvé successivement défini comme "barèmes de correction", "écoles de pensée sur ce qui constitue un texte bien traduit du point de vue linguistique ", "façon d'envisager ou d'étudier un problème ", "tout ce qui n'est pas de la pratique", "renseignements de base pour ensuite pouvoir appliquer les choses apprises ». Incertitude, donc, sinon mystère, aux yeux des répondants.

Notre objet, dans ce court article, n'est pas de proposer à notre tour des définitions du mot " théorie " (ou même de l'expression " théorie de la traduction "), et en ce qui le concerne il suffit de noter qu'aux yeux de Legrand $(1972: 256)$, elle constitue, soit un ensemble de règles et de conseils en vue de l'action ("théorie révolutionnaire"), soit un groupe plus ou moins lâche d'hypothèses sur un sujet contrôlable ou non. En effet, ce qui engage certains à se demander " À quoi sert la théorie? ", sinon à décréter d'emblée qu'elle est inutile, n'est pas la diffculté de définir intrinsèquement ce concept, mais bien l'automatisme fréquent de la tendance à ne l'envisager que par opposition à la pratique, et à lui attribuer par voie de conséquence un sens péjoratif. Or, c'est précisément ce caractère antinomique qui nous semble contestable, au moins sur le plan didactique : théorie et pratique de la traduction sont complémentaires et l'on ne peut pas dire d'une traduction qu'elle est "bonne" ou "mauvaise " à moins d'avoir une idée claire de ce qu'est l'équivalence.

$\mathrm{Si}$ " théorie de la traduction " est entendue au sens propre, c'est-à-dire comme étant une réflexion théorique sur la traduction, prise en tant qu'objet d'étude, on ne saurait en exiger qu'elle transforme un novice de la traduction en un professionnel, ni un mauvais traducteur en un bon, car ce ne sont pas là ses objectifs. Elle ne dotera pas non plus un traducteur d'une plus grande sensibilité à l'égard des mots et expressions de sa langue ou d'une langue étrangère. C'est pourquoi Kelly $(1979: 219)$ a raison d'écrire : "Had translation depended on its survival on theory, it would have died out long before Cicero. "

L'" utilité " de la théorie de la traduction est ailleurs. Les aspects de l'intérêt qu'elle peut présenter pour l'étudiant assez avancé, pour le pédagogue, et pour le traducteur sont nombreux, et ne feront pas ici l'objet d'une analyse de détail. Nous nous limiterons à souligner pour l'instant que seule la théorie de la traduction fournit la réflexion globale et fondamentale nécessaire pour mieux comprendre et systématiser le processus même de la traduction; qu'elle permet à l'étudiant, comme au spécialiste, de saisir plus facilement la spécificité de la traduction et d'entretenir à son sujet le discours indispensable pour rendre compte de leur propre activité ; qu'elle leur fournit enfin nombre de principes qui guideront leurs choix lors des opérations de traduction.

Malgré les aspects « utilitaires " que peut présenter la théorie, on peut considérer avec Holmes (1978: 60) que la nécessité de venir en aide au traducteur n'est pas le critère essentiel à retenir pour en justifier l'enseignement. Ce n'est pas pour devenir artiste, musicien ou romancier que certains poursuivent des recherches approfondies en histoire de l'art, en musicologie ou en littérature. De même, la théorie de la traduction n'a pas nécesssairement pour but d'aider explicitement l'étudiant ou le praticien. Au minimum, la théorie de la traduction fournit des éléments relativement à de nombreux aspects de la traduction qui, autrement, pourraient rester bien longtemps sans appréciation satisfaisante.

ROBERT LAROSE

\section{Notes}

- Nous tenons à remercier notre collegue et ami M. Jean Orsoni pour ses précieux commentaires.

1. La désignation d'un de ces cours, "Introduction to the Theory of Translation ", est assez surprenante alors qu'il est enseigné à la fin d'un programme d'études, sauf à croire que l'enseignement de cette matière se poursuit au deuxième cycle.

\section{RÉFÉRENCES}

DARBELNET, J. (1977) : "La spécialisation et l'enseignement de la traduction ", la Traduction. une profession, Montréal, Actes du VIII ${ }^{e}$ Congrès mondial de la Fédération internationale des traducteurs.

HOLMES, J.S. (1978) : "Translation Theory, Translation Studies, and the Translator ", la Traduction, une profession, Montréal, Actes du 
VIII' Congrès mondial de la Fédération internationale des traducteurs.

KELLY. G. (1979) : The True Interpreter : A History of Translation Theory and Practice in the West, New York, St. Martin's Press.

LEGRAND, G. (1972) : Dictionnaire de philosophie, Paris, Bordas. 